\title{
A Frequency Domain Equalization Algorithm for Fast Time-Varying Fading Channels
}

\author{
Le-Nam Tran, Een-Kee Hong, and Huaping Liu
}

\begin{abstract}
Conventional frequency domain equalization (FDE) schemes were originally devised for quasi-static channels. Thus, such equalization schemes could suffer from significant performance degradation in fast-fading channels. This paper proposes a frequency domain equalization algorithm to mitigate the effect of fast time-varying fading. First, a mathematical expression is derived to quantify the total interference resulting from the time variation of the channel. Then, the proposed approach attempts to eliminate the effect of time-variations of the channel. This cancellation allows efficient use of the classical FDE structures in fast time-varying fading environments, although they are built upon the quasi-static channel model. Simulation results of bit-error-rate performance are provided to demonstrate the effectiveness of the proposed algorithm.
\end{abstract}

Index Terms: Cancellation, direct sequence code division multiple access (DS-CDMA), fast time-varying fading channels, frequency domain equalization (FDE).

\section{INTRODUCTION}

The next-generation mobile communication systems are expected to support high data rates that usually require a bandwidth larger than the coherence bandwidth of the channel, resulting severe frequency-selective fading channels. In turn, the intersymbol interference (ISI) caused by severe frequency selectivity of the channel could limit the maximum transmission rate. As the time dispersion of the radio channel spans over a number of symbol durations, the design for high-speed reliable communications becomes challenging since the traditional antimultipath approaches such as multipath-diversity combining become inefficient to recover the transmitted signals [1].

To cope with severe frequency selectivity, the entire signal bandwidth could be divided into multiple sub-bands, each of which is narrow enough to assume a frequency-flat fading. This concept leads to the multi-carrier techniques, in particular, the orthogonal frequency-division multiplexing (OFDM) technique

Manuscript received April 15, 2008; approved for publication by Kwang Soon Kim, Division II Editor, February 24, 2009.

This work was partly supported by the IT R\&D program of IITA [2008-F-00502, Game Theoretic Approach for Cross-layer Design in Wireless Communications] and the MKE (The Ministry of Knowledge Economy), Korea, under the ITRC (Information Technology Research Center) support program supervised by the IITA (Institute for Information Technology Advancement) (IITA-2009C1090-0903-0011).

L.-N. Tran and E.-K. Hong are with the School of Electronics and Information, Kyung Hee University, South Korea, email: \{nltran, ekhong\} @khu.ac.kr.

H. Liu is with the School of Electronic Engineering and Computer Science, Oregon State University, U.S.A., email: hliu@eecs.oregonstate.edu.

The authors wish to thank Dr. Jack Holtzman and Peter Gaal from QUALCOMM. INC. for helpful discussions on methods to evaluate the performance of the proposed algorithm, especially for the valuable suggestion on the practical channel estimation methods.
[2]. However, OFDM also has many disadvantages such as a high sensitivity to frequency offset, and to nonlinear distortion which requires expensive linear amplifiers due to the high peakto-average-power ratio (PAPR) of the transmitted signals.

An alternative solution to deal with frequency selectivity is to equalize the received signals. Equalization could be accomplished in either the time domain or the frequency domain. Generally, the classical time domain equalization (TDE) has a high computational complexity if the channel delay spread is large. The complexity of TDE can be lowered by transforming its operation in time domain to frequency domain. Although the concept of frequency domain equalization (FDE) appeared almost three decades ago [3], single-carrier frequency domain equalization (SC-FDE) has been investigated only recently as a promising approach for broadband wireless communications [4].

Basically, FDE was developed based on the correspondence between the circular convolution of two sequences in the time domain and the point-wise product of their DFT outputs in the frequency domain [5]. If the transmitted data are designed to be periodic, the received signals are the circular convolution between the transmitted signal and the channel impulse response (CIR). To maintain circular convolution, in addition to the cyclic data transmission format, the channel must be quasi-static, that is, the path gains are constant over a block interval. Most of present FDE structures are based on the quasi-static fading channel. The problem of FDE in time varying channels has been treated sparsely in different directions. For example, using the basis extension model (BEM), the authors in [6] presented iterative FDE with widely linear filtering. In [7], the windowing technique was utilized to transform the channel matrix into a banded form. Based on this simplified model, the iterative equalization was applied. However, the optimal window design is complicated.

In this paper, rather than simplifying the channel matrix for efficient matrix inversion, we propose an equalization algorithm to mitigate the effect of time variations in fast time-varying fading channels. First, we derive a mathematical model to characterize the interference caused by time-variations of the channel. As shown later, this interference depends on both the transmitted data and CIR. Thus, the suppression of this interference is only possible if the transmitted data and CIR are available at the receiver. In practice, the channel can be estimated, typically by using the transmitted pilot signals, but the transmitted data cannot be known in advance. Hence, our equalization algorithm is performed through several steps. In the first step, the tentative estimates of the transmitted data are obtained by using the conventional FDE. From the second step, the interference caused by the time-variation of the channel is eliminated based on tentative estimates from the first step and the estimated CIR. Con- 
ventional FDE is then applied again to the resulting signals. The proposed method can be applied to any conventional communication systems, but we will focus on direct sequence code division multiple access (DS-CDMA) systems in our analysis and numerical examples as a study case.

The rest of this paper is organized as follows. Section II briefly reviews the background of FDE and their applications to DS-CDMA systems. The analysis of the effect of time-varying multipath fading channels on FDE and description of the proposed equalization algorithm are presented in Section III. The performances of our proposed approach are evaluated with computer simulations in Section IV, followed by conclusions in Section V.

\section{BACKGROUND OF FREQUENCY DOMAIN EQUALIZATION}

FDE has been investigated as one of the promising approaches for the CDMA-based 3G systems and beyond [8], [9] since the RAKE combining technique [1] exhibits poor performance in severe frequency selective fading channels. Before analyzing FDE in time-varying channels, we first briefly review the conventional FDE for quasi-static channels. For simplicity and convenient illustration purpose, we are interested in the frequency domain liner equalization for DS-CDMA systems as an example. It means that the proposed equalization algorithm which will be described in the next section is applicable to all wireless communications systems, including other equalization structures (e.g., hybrid decision feedback equalization (HDFE) [5], [10], [11]) and non-spread spectrum systems.

\section{A. Frequency Domain Linear Equalization (FD-LE) for DS- CDMA Systems}

The general FD-LE for DS-CDMA applications is illustrated in Fig. 1. At the transmitter, a block of $M$ data modulated symbols $\left\{d_{n}\right\}$ is spread by a spreading sequence $c_{m}$ with a processing gain $S F$ to produce the spread signals $s_{n}$. Then, a known sequence of $L$ chips is added to the spread signals to form a transmitted data block of size $P=M \times S F+L$ chips. This data extension known as unique word (UW) extension [5] is to make the transmitted data periodic, and thus the channel matrix become circulant. Another type of data extension that can play the same role is cyclic prefix extension [10]. However, in this paper, we employ UW extension because it is beneficial for channel estimation. The received signals are written as

$$
r_{n}=\sum_{l=0}^{\rho-1} h_{l} s_{n-l}+\eta_{n}, n=0,1, \cdots, P-1
$$

where $h_{l}, l=0,1, \cdots \rho-1$ denotes the fading coefficient of the $(l+1)$ th path, $\eta_{n}$ is the zero-mean complex white Gaussian noise with variance $\sigma_{n}^{2}$. In (1), the channel is assumed to be quasistatic (block fading model). Under the condition that $L \geq \rho$, we can express the frequency domain equivalence of (1) as

$$
R_{k}=H_{k} S_{k}+N_{k}, k=0,1, \cdots, P-1
$$

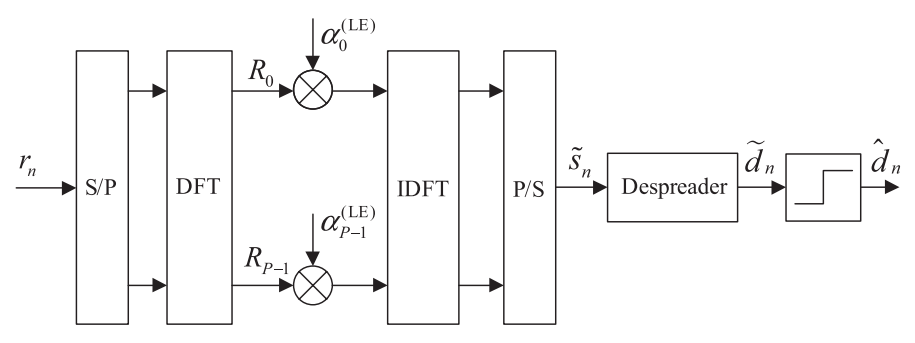

Fig. 1. FD-LE for DS-CDMA systems.

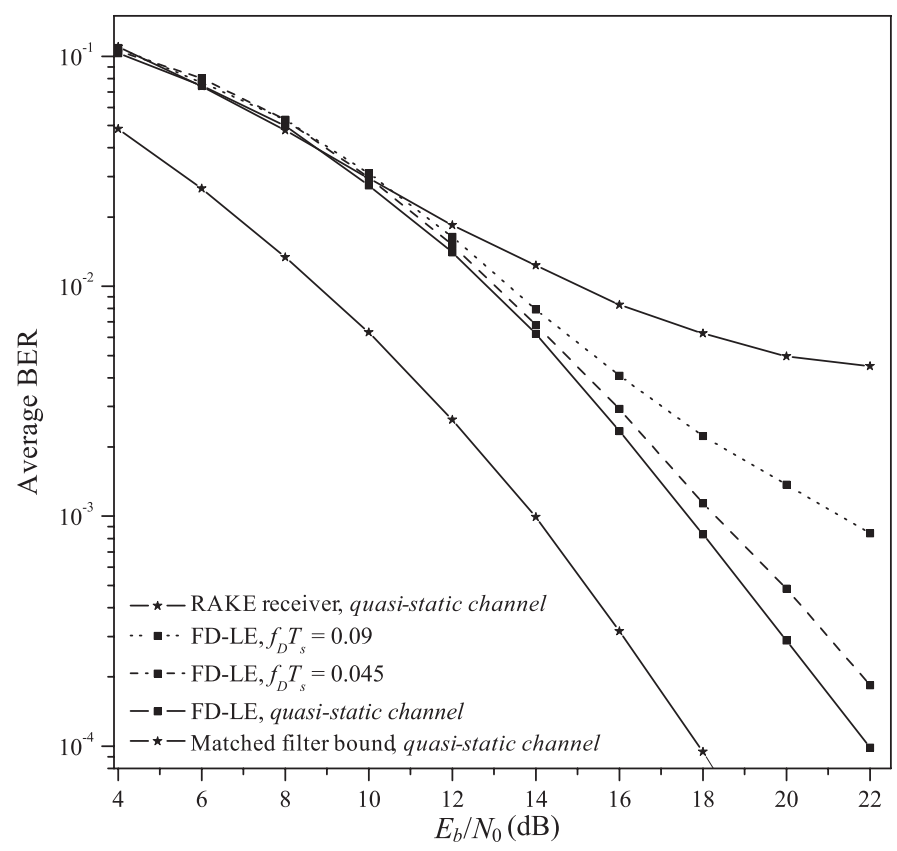

Fig. 2. Performance of FD-LE in quasi-static and time-varying fading channels.

where

$$
\begin{aligned}
R_{k} & =\sum_{l=0}^{P-1} r_{n} e^{-j 2 \pi k n / P}, \\
H_{k} & =\sum_{l=0}^{\rho-1} h_{l} e^{-j 2 \pi k l / P} \\
N_{k} & =\sum_{n=0}^{P-1} \eta_{n} e^{-j 2 \pi k n / P}
\end{aligned}
$$

are the results of applying a $P$-point DFT to the received signals, CIR and additive noise, respectively. It is important to mention that (2) holds only in quasi-static channels.

The $P$-point DFT outputs of the received signals $\left\{R_{k}\right\}$ are weighted with linear equalizer coefficients $\left\{\alpha_{k}^{(\mathrm{LE})}\right\}$. The resulting signals are transformed back into the time domain by a $P$ point IDFT block. If the minimum mean-square error (MMSE) criterion is used, the FD-LE coefficients can be easily found as [8], [9]

$$
\alpha_{k}^{(\mathrm{LE})}=\frac{H_{k}^{*}}{\left|H_{k}\right|^{2}+\sigma_{n}^{2}}, k=0,1, \cdots, P-1 .
$$

The equalized chips $\tilde{s}_{n}$ are passed through the despreader, followed by a threshold detector to detect the transmitted data. 


\section{B. Impact of Channel Variations on Performance of FDE}

In this section, we provide the simulation results obtained with the FD-LE structure to demonstrate the impact of channel time-variations on the BER performance when the user speed increases. The detailed simulation parameters are given in Section IV.

Fig. 2 shows the BER of various anti-multipath approaches for quasi-static and time-varying channels. For RAKE combining, we consider RAKE combiner of 2 fingers. In quasi-static fading channels, while RAKE combining technique is not reliable due to the severe frequency selectivity of the channel, FD-LE achieves excellent performance. It is observed that the performances of FD-LE in time-varying channels are significantly deteriorated as the user speed increases. When the user speed $v$ is $60 \mathrm{~km} / \mathrm{h}$, the maximum Doppler spread $f_{D}$ is $167 \mathrm{~Hz}$, producing a normalized Doppler frequency change (defined as the product of the block duration $T_{\text {block }}$ and Doppler frequency $f_{D}$ ) of 0.045 . This degree of mobility represents a slowly timevarying channel. Thus, the traditional FDE is still efficient to combat multipath distortion. However, the BER performance exhibits an error floor as $v$ reaches to $120 \mathrm{~km} / \mathrm{h}$. In this case, the maximum Doppler spread is doubled and the normalized Doppler frequency becomes 0.09. This channel can be viewed as a fast time-varying channel [12], and the path gains are not constant over a data block.

\section{PROPOSED FDE ALGORITHM FOR TIME-VARYING FADING CHANNELS}

The FD-LE [8], [9] presented in Section II and many other traditional FDE structures (e.g., HDFE in [5], [10], [11]) were developed based on the assumption of quasi-static channel. When the data block duration is longer than the channel coherence time, the channel gain cannot be considered constant over one block. Consequently, FDE algorithms derived based on (2) will suffer from significant performance degradation in fast timevarying channels.

For OFDM systems, time-varying fading causes a loss of subcarrier orthogonality, which results in inter-carrier interference (ICI). While the impact of ICI caused by channel variations on the performance of OFDM systems has been studied extensively [12]-[17], data transmission in time-varying channels with FDE has received limited attention. In this section, we mathematically analyze the effect of channel time-variations on the performance of FDE schemes. Based on such analysis, we develop a equalization scheme to improve the performance of FDE in time-varying channels.

\section{A. Analysis of the Impact of Time-Varying Fading Channels on Classical FDE}

For multipath time-varying channel model, the received signal is written by

$$
r_{n}=\sum_{l=0}^{\rho-1} h_{l}(n) s_{n-l}+\eta_{n}
$$

An $P$-point DFT is applied to $\left\{r_{n}\right\}$ to transform the received signals into the frequency domain. To simplify notation, we only consider the mathematical expression for a certain $k$ th frequency bin of the received signal in our analysis, which is given by

$$
\begin{aligned}
R_{k} & =\sum_{n=0}^{P-1} r_{n} e^{-j 2 \pi k n / P} \\
& =\sum_{n=0}^{P-1} \sum_{l=0}^{\rho-1} h_{l}(n) s_{n-l} e^{-j 2 \pi k n / P}+\sum_{n=0}^{P-1} \eta_{n} e^{-j 2 \pi k n / P} \text {.(5) }
\end{aligned}
$$

For each block, we can decompose $h_{l}(n)$ into two components, a time-invariant part and a time-variant part, as

$$
h_{l}(n)=\omega_{l}+\psi_{l}(n)
$$

where

- $\omega_{l}$ denotes the average channel gain of the $l$ th path within one block. This term represents the constant (time-invariant) component of CIR in one data block. If $P$ is the block size, $\omega_{l}$ can be written by

$$
\omega_{l}=\frac{\sum_{n=1}^{P} h_{l}(n)}{P} .
$$

- $\psi_{l}(n)$ is the difference between channel coefficient at time $n$ and the average value $\omega_{l}$ of the $l$ th path. This value measures the deviation of the channel coefficient at time $n$ from the average value $\omega_{l}$. In other words, $\psi_{l}(n)$ measures the degree of change of the CIR. Note that, $\psi_{l}(n)$ can be computed using (6) as $\psi_{l}(n)=h_{l}(n)-\omega_{l}$ if the estimates of $h_{l}(n)$ are available.

From (5) and (6), the received signal in the frequency domain can be written by

$$
\begin{aligned}
R_{k}= & \underbrace{\sum_{n=0}^{P-1} \sum_{l=0}^{\rho-1}\left\{\omega_{l} s_{n-l}+\eta_{n}\right\} e^{-j 2 \pi k n / P}}_{\Omega_{k}} \\
& +\underbrace{\sum_{n=0}^{P-1} \sum_{l=0}^{\rho-1} \psi_{l}(n) s_{n-l} e^{-j 2 \pi k n / P}}_{\Delta_{k}} .
\end{aligned}
$$

The first term of the right-hand side of (8), $\Omega_{k}$, can be viewed as the frequency response of the received signal in quasi-static channels. According to (2), we can simplify $\Omega_{k}$ as

$$
\Omega_{k}=H_{k} S_{k}+N_{k}, k=0,1, \cdots, P-1
$$

where $H_{k}=\sum_{l=0}^{\rho-1} \omega_{l} e^{-j 2 \pi k l / P}$ and $N_{k}=\sum_{n=0}^{P-1} \eta_{n} e^{-j 2 \pi k n / P}$ are the DFTs of the average value of CIR and additive noise, respectively. Let us define

$$
\Psi_{k}^{(l)}=\sum_{n=0}^{P-1} \psi_{l}(n) s_{n-l} e^{-j 2 \pi k n / P}
$$

which is the DFT of the product of the two sequences $\left\{\psi_{l}(n)\right\}$ and $\left\{s_{n-l}\right\}$. The second term $\Delta_{k}$ in (8) can be written by

$$
\Delta_{k}=\sum_{l=0}^{\rho-1} \sum_{n=0}^{P-1} \psi_{l}(n) s_{n-l} e^{-j 2 \pi k n / P}=\sum_{l=0}^{\rho-1} \Psi_{k}^{(l)}
$$


resulting in a more compact form of (5) which is expressed as

$$
R_{k}=\underbrace{H_{k} S_{k}+N_{k}}_{\Omega_{k}}+\underbrace{\sum_{l=0}^{\rho-1} \Psi_{k}^{(l)}}_{\Delta_{k}}, k=0,1, \cdots, P-1 .
$$

In (12), $\Delta_{k}$ is considered as the interference resulting from the channel variation. If the channel is assumed to be constant within one block period (i.e., $\left.\psi_{l}(n)=0, \forall n\right), \Delta_{k}$ will vanish. For time-varying channels, $\Delta_{k}$ destroys the corresponding equivalency between circular convolution in the time domain and multiplication in the frequency domain, and thus degrades the performance of FDE. The value of $\Delta_{k}$ is dependent on the severity of the channel fluctuation; $\Delta_{k}$ increases as the mobile speed increases.

Our goal is to eliminate this interference, but this requires $a$ priori knowledge of the transmitted signals $s_{n}$, as well as the channel coefficients. In the following, we propose an equalization algorithm which is accomplished through several steps to reduce the effect of time variations of the channel.

\section{B. The Proposed FDE Algorithm}

For time-varying channels, FDE is not conceptually applicable due to the presence of $\Delta_{k}$ in (12), since the frequency response of the received signal is no longer equal to the point-wise product of the frequency responses of the transmitted signal and the CIR. It is desirable to cancel $\Delta_{k}$ so that FDE can be efficiently exploited. From (11), the computation of $\Delta_{k}$ requires the followings:

- The values of $\psi_{l}(n)$ : These values can be obtained through channel estimation which generates estimates $\hat{h}_{l}(n)$. An estimate of $\psi_{l}(n)$ is then given by

$$
\hat{\psi}_{l}(n)=\hat{h}_{l}(n)-\hat{\omega}_{l}
$$

where the average channel gain $\hat{\omega}_{l}$ can be estimated using (7).

- The values of the transmitted sequence $s_{n-l}$ : In practice, these values are not known at the receiver in advance. However, we can make use of the tentative signal estimates that are obtained by applying conventional FDE algorithms to the received signals over time-varying channel, ignoring the effect of time-variant components.

Our proposed method is carried out through several steps as follows:

- Step 1: Apply FDE for time-varying channels to yield tentative symbol estimates.

Any existing FDE structures (e.g., FD-LE in Fig. 1 or HDFE in [11]) can be employed.

- Step 2: Compute $\hat{\Delta}_{k}$ from the tentative symbol estimates obtained in step 1.

$$
\begin{aligned}
\hat{\Psi}_{k}^{(l)} & =\sum_{n=0}^{P-1} \hat{\psi}_{l}(n) \hat{s}_{n-l} e^{-j 2 \pi k n / P}, l=0,1, \cdots, \rho-1 \\
\hat{\Delta}_{k} & =\sum_{l=0}^{\rho-1} \hat{\Psi}_{k}^{(l)} .
\end{aligned}
$$

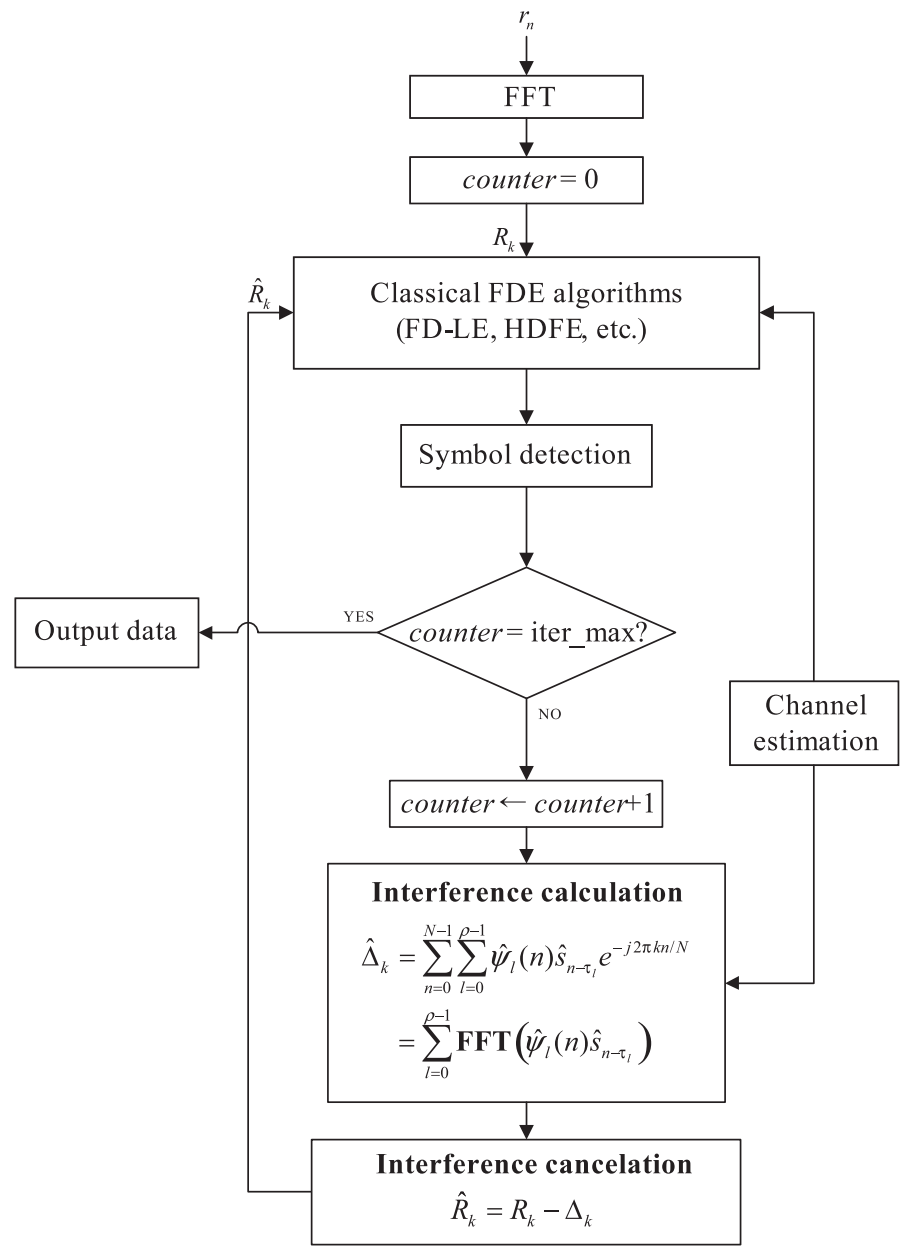

Fig. 3. Signal processing flow of proposed FDE for time-varying channels.

- Step 3: Subtract $\hat{\Delta}_{k}$ from $R_{k}$ in (12), which yields

$$
\hat{R}_{k}=R_{k}-\hat{\Delta}_{k}
$$

If the estimate of $\Delta_{k}$ is perfect, $\hat{R}_{k}$ is equal to $\Omega_{k}$. Since perfect data detection in step 2 is impossible, only part of the total interference will be removed.

- Step 4: Apply FDE to $\hat{R}_{k}$ computed in step 3 to obtain symbol estimates again.

This step is very similar to step 1 . Because the impact of $\Delta_{k}$ is partially reduced, better symbol estimates can be achieved. Therefore, the overall performance of FDE is improved. The signal processing flow of steps from 1 to 4 is depicted in Fig. 3.

We also notice that the number of phases in the proposed algorithm can be increased by applying the turbo principle [18]. Namely, steps 2 to 4 can be repeatedly carried out to further enhance the performance. The method used in [19] can be helpful to account for the improved reliability of signal detection after each phase of the proposed algorithm. However, such a method requires high computational complexity, and thus is not considered in this paper. 
Table 1. Taped-delay-line parameters for vehicular environment.

\begin{tabular}{cccc}
\hline Tap & Delay $(\mathrm{ns})$ & Average power & Doppler spectrum \\
\hline 1 & 0 & -2.5 & Classic \\
\hline 2 & 271 & 0 & Classic \\
\hline 3 & 8943 & -12.8 & Classic \\
\hline 4 & 13008 & -10.0 & Classic \\
\hline 5 & 17073 & -25.2 & Classic \\
\hline 6 & 20054 & -16.0 & Classic \\
\hline
\end{tabular}

Table 2. Simulation parameters.

\begin{tabular}{cc}
\hline Parameters & Selected values \\
\hline FFT size & 1024 \\
\hline Spreading factor & 16 \\
\hline Modulation & QPSK \\
\hline Carrier frequency & $3 \mathrm{GHz}$ \\
\hline
\end{tabular}

\section{PERFORMANCE COMPARISON}

In this section, we evaluate the performance of the proposed algorithm for FDE in time-varying channels to demonstrate the effectiveness of the proposed algorithm to mitigate the effect of fast time-varying fading. We also examine the robustness of the proposed algorithm with practical channel estimation. Although we focus on DS-CDMA applications as a case study, the proposed algorithm can be applied to most wireless communication systems.

\section{A. System Parameters}

The channel B of the vehicular environment as described in Recommendation ITU-R M.1225 [20] is considered. The chip rate is selected to be $3.6864 \mathrm{Mbps}$ as in CDMA2000. The delay between multipaths is assumed to be an integer multiple of a chip interval ( $1 / 3.6864 \mathrm{Mcps} \approx 271 \mathrm{~ns})$, so a modified version of the channel model is used. The power delay profile for the six independent paths of the modified channel B is listed in Table 1.

We consider the performance of an uncoded DS-CDMA system using QPSK modulation. A 16-ary Walsh code is used for spreading. The FFT size is 1024; thus the number of symbols (including unique word extension) in one transmission block is $1024 / 16=64$ symbols. Consequently, the block duration is $T_{\text {block }}=0.28 \mathrm{~ms}$. The maximum Doppler shift is calculated assuming a carrier frequency of $3 \mathrm{GHz}$. Simulation parameters are summarized in Table 2. The channel simulator is built based on the JTC channel model in [21].

\section{B. Simulation Results with Ideal Channel Estimation}

The UW length for FDE is required to be longer than the length of the CIR. The modified channel model in Table 1 has an excess delay spanning $20054 / 271=74$ chips. The UW of 80 chips selected from a PN sequence is inserted into each data block. The performances of FD-LE combined with the proposed equalization algorithm and the conventional FD-LE (i.e., FD-LE without canceling the effect of channel variation) are compared in Fig. 4.

In Fig. 4, the legend 'no cancellation' denotes the system performance when the conventional FD-LE is applied to timevarying channels, and 'practical cancellation' represents the

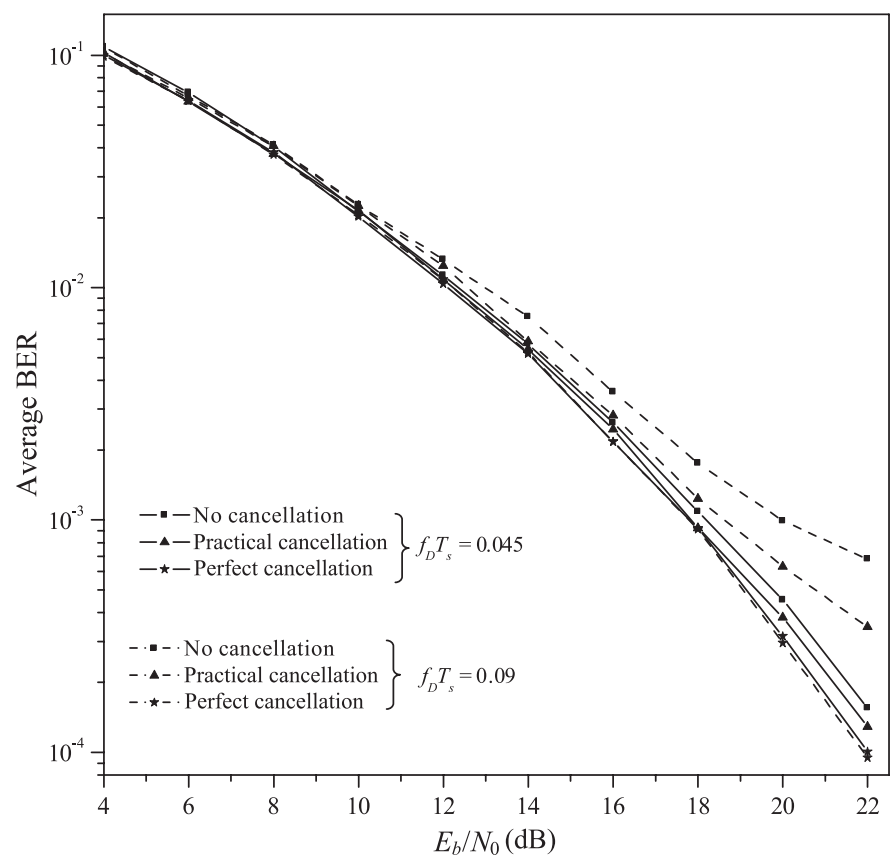

Fig. 4. Performance of the proposed algorithm applied to FD-LE with ideal channel estimation, UW $=80$ chips.

performance of the proposed algorithm scheme where the cancellation is performed with practical data detection (detected data from the first step). For comparison purpose, we also consider the ideal case labeled by 'perfect cancellation,' in which the channel coefficients and the transmitted data are known a priori at the receiver. In this ideal case, the interference term can be reconstructed accurately and cancelled perfectly. Accordingly, we can infer from Fig. 4 that the performance of the proposed FDE in this ideal case is independent of Doppler frequency, and is equal to that of traditional FDE applied to quasi-static channel. It is also noted that a higher performance gain of the proposed equalization over the conventional FD-LE can be achieved as the user mobility increases and the proposed algorithm can eliminate the error floor. When the normalized Doppler frequency is 0.09 , a performance gain of about $2.2 \mathrm{~dB}$ is observed between between 'no cancellation' and 'practical cancellation' at the BER $=7 \times 10^{-4}$.

\section{Simulation Results with Practical Channel Estimation}

\section{C.1 Channel Estimation}

In the proposed algorithm, the channel gain (fading coefficient) at every data symbols within one block must be known. An additional benefit of UW is that it can be used as pilot signals to estimate the channel. The time-varying channel estimation in our work is performed through two steps. First, the channel gains at UW positions are estimated using the multi-stage serial interference cancellation approach (M-SIC) as described in [22]. Second, the channel gains at the data field are obtained by interpolating the estimated channel coefficients at the UW positions. To ensure an accurate estimation using the M-SIC approach, the UW length is increased to 160 chips (a little more than twice of the channel length) at the cost of a lower bandwidth efficiency. A comprehensive study of interpolation method for channel es- 


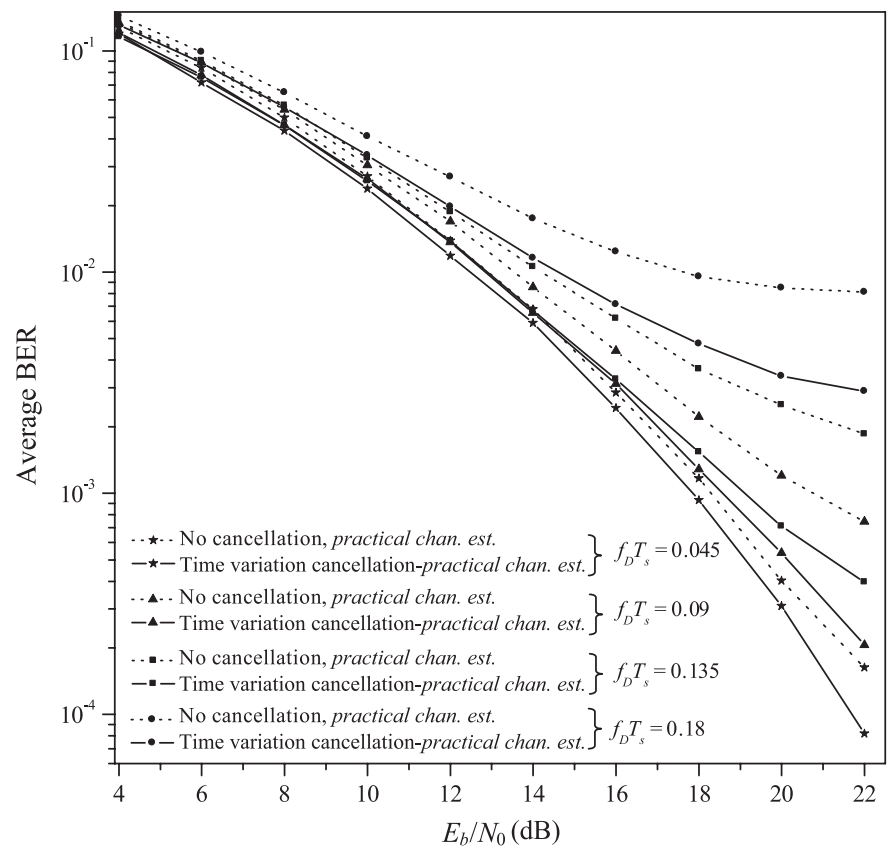

Fig. 5. Performance of the proposed algorithm applied to FD-LE with practical channel estimation (UW $=160$ chips), and different degrees of mobility.

timation can be found in [23]. When computing the channel estimates at the data field of each block, we use the estimated channel gains at the UW position of the previous and current blocks. The estimated channel gains at the data fields are given by using a simplified sinc interpolation as below

$$
\hat{h}(l, n)=\sum_{i=1}^{2} \hat{h}\left(l, n_{i}\right) \frac{\sin \left(2 \pi f_{D} T_{c}\left(n_{i}-n\right)\right)}{2 \pi f_{D} T_{c}\left(n_{i}-n\right)}
$$

where $T_{c}$ is the chip duration, and $f_{D}$ is the maximum Doppler frequency. For the mobile speed estimation, the simple method in [24] can be employed. In this paper, the value of mobile speed is assumed to be known at the receiver. In (16), $\hat{h}\left(l, n_{i}\right)$ is the estimated channel gain of the $l$ th path at the UW portion, $n_{i}$ indicates the center position of the $i$ th $\mathrm{UW}$, and $n$ is the position of each chip in the block.

\section{C.2 Performance of the Proposed Algorithm with Practical Channel Estimation in Time Varying Channel}

Fig. 5 plots the BER performances of the proposed equalization algorithm with practical channel estimation in time varying channels. Several degrees of mobility are considered where the normalized Doppler frequency is up to $0.18(v=240 \mathrm{~km} / \mathrm{h})$. It is observed that the proposed algorithm provides a remarkable gain over the traditional method with practical channel estimation in high mobility scenarios.

\section{C.3 Impact of Spreading Factor}

In Fig. 6, we evaluate the proposed equalization algorithm with different values of spreading factor (SF) to investigate the effect of SF on the proposed algorithm. The normalized Doppler frequency is set to be $0.09(v=120 \mathrm{~km} / \mathrm{h})$. Although our approach always outperforms the traditional FDE scheme, its per-

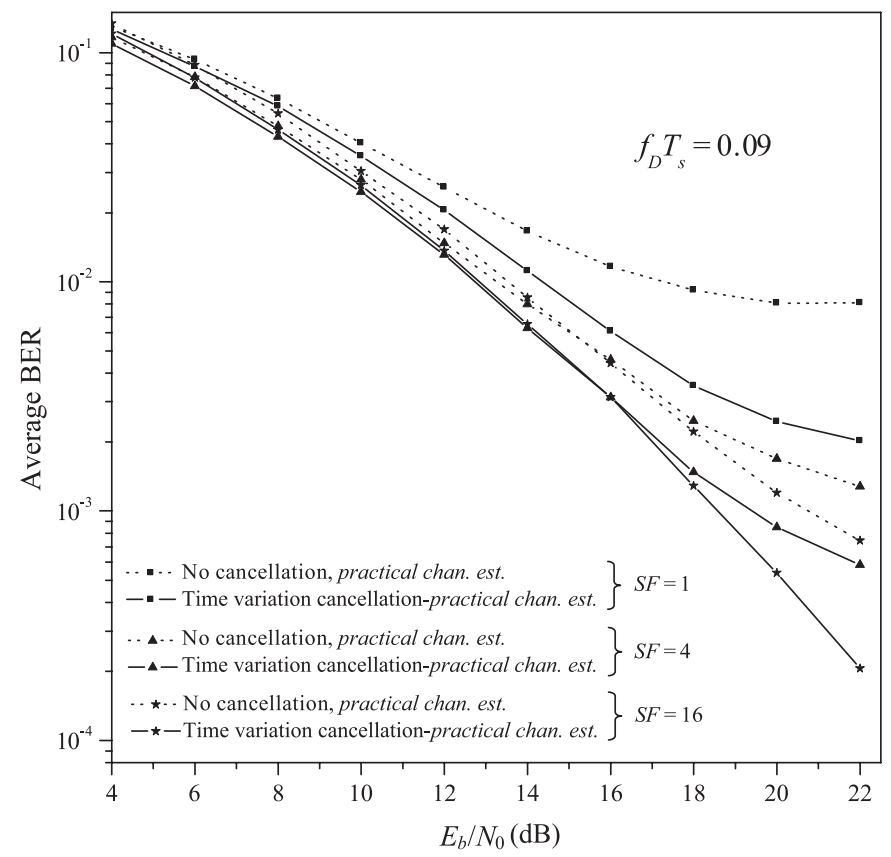

Fig. 6. Performance of the proposed algorithm applied to FD-LE with practical channel estimation $\left(f_{D} T_{s}=0.09, \mathrm{UW}=160\right.$ chips), and different values of spreading factor (SF).

formance is degraged as the spreading factor becomes small. This is understandable and can be explained as follows. For FDE, the equalization is performed in frequency domain and detection is performed in time domain. The interference expressed in (11) depends on time-varying coefficients and detected chips. The accuracy of detected chips in time domain detection relies on the spreading factor. Namely, a small value of SF results in less accuracy of detected chip, and thus the interference is not correctly recovered. This eventually causes a performance degradation on the performance of the proposed method.

\section{CONCLUSIONS}

We have proposed an effective algorithm to cancel the timevariant component of the received signals for robust operation of SC-FDE over fast time-varying fading channels. We first derived the mathematical expressions to model the effect of channel time-variations on the performance of FDE and then provided the multi-step equalization scheme. The proposed scheme improves the performance of conventional FDE by canceling the effect of fast time-varying fading channel. In the proposed scheme, the tentative data estimates are obtained with the conventional FDE and then the interference term caused by the time-variation of the channel is quantified and canceled, followed by further equalization on the signal after interference cancellation.

To demonstrate the robustness of our proposed algorithm, we have evaluated the performance of the proposed interference cancellation scheme combined with the existing FD-LE scheme in different time-varying fading channels by computer simulations, where the performance with perfect interference cancellation is used as the baseline performance. For relatively high Doppler spreads, the interference cancellation method can pro- 
vide about $2.2 \mathrm{~dB}$ gain over the traditional FD-LE algorithms without the cancellation of time variation components at a BER of $7 \times 10^{-4}$. The multi-step FDE algorithm proposed in this paper is a promising solution to achieve robust performance in time varying fading environments since it can reduce the irreducible error floor that appears in traditional FDE schemes.

\section{REFERENCES}

[1] J. G. Proakis, Digital Communications, 4th ed. McGraw Hill, 2001.

[2] J. A. C. Bingham, "Multicarrier modulation for data transmission: An idea whose time has come," IEEE Commun. Mag., vol. 28, pp. 5-14, May 1990.

[3] T. Walzman and M. Schwartz, "Automatic equalization using the discrete frequency domain," IEEE Trans. Inf. Theory, vol. 19, no. 1, pp. 59-68, Jan. 1973.

[4] Air Interface for Fixed Broadband Wireless Access Systems Part A: Systems between 2 and $11 \mathrm{GHz}$, IEEE Std. 802.16ab-01/01, 2001.

[5] N. Benvenuto and S. Tomasin, "On the comparison between OFDM and single carrier modulation with a dfe using a frequency domain feedforward filter," IEEE Trans. Commun., vol. 50, no. 6, pp. 947-955, June 2002.

[6] B. K. Ng and D. Falconer, "A novel frequency domain equalization method for single-carrier wireless transmissions over doubly-selective fading channels," in Proc. IEEE GLOBECOM, vol. 1, Nov. 2004, pp. 237241 .

[7] P. Schniter and H. Liu, "Iterative frequency-domain equalization for single-carrier systems in doubly-dispersive channels," in Proc. IEEE AC$S S C$, vol. 1, Nov. 2004, pp. 667-671.

[8] K. L. Baum, T. A. Thomas, F. W. Vook, and V. Nangia, "Cyclic-prefix: An improved transmission method for broadband DS-CDMA cellular systems," in Proc. IEEE WCNC, vol. 1, May 2002, pp. 183-188.

[9] F. Adachi, T. Sao, and T. Itagaki, "Performance of multicode DS-CDMA using frequency domain equalization in frequency selective fading channel," Electron. Lett., vol. 39, no. 2, pp. 239-241, Jan. 2003.

[10] D. Falconer, S. L. Ariyavisitakul, A. Benyamin-seeyar, and B. Eidson, "Frequency domain equalization for single-carrier broadband wireless systems," IEEE Commun. Mag., no. 4, pp. 58-66, Apr. 2002.

[11] L. N. Tran, E. K. Hong, and H. Liu, "Two-stage hybrid decision feedback equalization for DS-CDMA systems," IEEE Trans. Wireless Commun., vol. 7, no. 5, pp. 1490-1494, May 2008.

[12] W. G. Jeon, K. H. Chang, and Y. S. Cho, "An equalization technique for orthogonal frequency-division multiplexing systems in time-variant multipath channels," IEEE Trans. Commun., vol. 47, no. 1, pp. 27-32, Jan. 1999.

[13] A. Stamoulis, S. N. Diggavi, and N. Al-Dhahir, "Intercarrier interference in MIMO OFDM," IEEE Trans. Signal Process., vol. 50, no. 10, pp. 24512464 , Oct. 2002.

[14] X. Cai and G. B. Giannakis, "Bounding performance and suppressing intercarrier interference in wireless mobile OFDM," IEEE Trans. Commun., vol. 51, no. 12, pp. 2047-2056, Dec. 2003.

[15] A. Gorokhov and J. P. Linnartz, "Robust OFDM receivers for dispersive time-varying channels: equalization and channel acquisition," IEEE Trans. Commun., vol. 52, no. 4, pp. 572-583, Apr. 2004.

[16] P. Schniter, "Low-complexity equalization of OFDM in doubly selective channels," IEEE Trans. Signal Process., vol. 52, no. 4, pp. 1002-1011, Apr. 2004.

[17] S. Tomasin, A. Gorokhov, H. Yang, and J.-P. Linnartz, "Iterative interference cancellation and channel estimation for mobile OFDM," IEEE Trans. Wireless Commun., vol. 4, no. 1, pp. 238-245, Jan. 2005.

[18] J. Hagenauer, "The turbo principle: Tutorial introduction and state of the art," in Proc. International Symposium on Turbo Codes, Brest, France, Sept. 1997, pp. 1-11.

[19] B. Ng, C. T. Lam, and D. Falconer, "Turbo frequency domain equalization for single-carrier broadband wireless systems," IEEE Trans. Wireless Commun., vol. 6, no. 2, pp. 759-767, Feb. 2007.

[20] Recommendation ITU-R M.1225, "Guideline for evaluation of radio transmission technologies for IMT-2000,” 1997, question ITU-R 39/8.
[21] Joint Technical Committee (Air) Standards Contribution, "Technical report on RF channel characterization and system deployment modeling," Tech. Rep., Sept. 1994, JTC(AIR)/94.09.23-065R6.

[22] E. Onggosanusi, A. Papasakellariou, and A. Dabak, "Derivation of channel estimation error model for CDMA EBM evaluation methodology," Tech. Rep., Dec. 2004, C30-20041206-022. [Online]. Available: ftp://ftp. 3gpp2.org/TSGC/ Working/2004/2004-12-Kauai/ TSG-C-2004-12-Kauai/ WG3

[23] G. Yue, X. Zhou, and X. Wang, "Performance comparisons of channel estimation techniques in multipath fading CDMA," IEEE Trans. Wireless Commun., vol. 3, no. 3, pp. 716-724, May 2004.

[24] L. Zhao and J. W. Mark, "Mobile speed estimation based on average fade slope duration," IEEE Trans. Commun., vol. 52, no. 12, pp. 2066-2069, Dec. 2004.

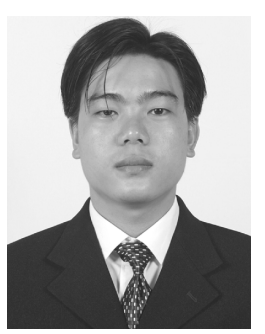

Le-Nam Tran received the B.S. degree in Electrical Engineering from Ho Chi Minh National University of Technology, Vietnam in 2003. Since 2004, he has been supported by "Korean Government IT Scholarship Program" to follow the M.S. degree in Kyung Hee University, Kyunggi, Korea, and received his M.S. Electrical Engineering in 2006. He is working toward the Ph.D. degree in electrical engineering at the same university. He received the Best Paper Award from IITA in August 2005. His current interests include mobile communication systems, equalization techniques, spread spectrum and CDMA schemes, multiuser MIMO systems.

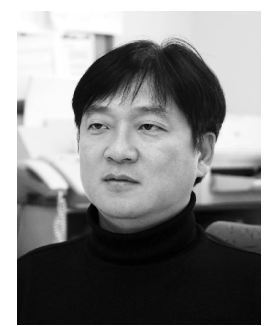

Een-Kee Hong received the B.S., M.S., and Ph.D. degrees, all in electrical engineering, from Yonsei University, in 1989, 1991, and 1995, respectively. He was senior research engineer at SK Telecom from September 1995 to February 1999 and visiting senior engineer at NTT DoCoMo from October 1997 to December 1998. He is currently Associate Professor at Kyung Hee University, South Korea. His research interests are in physical layer in wireless communication, spectrum engineering, and cross-layer optimization. He was awarded the Best Paper Award, Institute of Information Technology Assessment, and Haedong Best Paper Award, KICS in 2005.

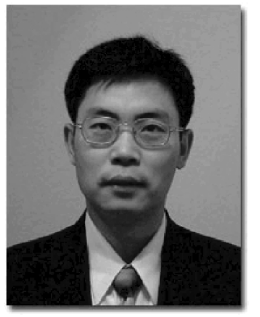

Huaping Liu received his B.S. and M.S. degrees from Nanjing University of Posts and Telecommunications, Nanjing, P. R. China, in 1987 and 1990, respectively, and his Ph.D. degree from New Jersey Institute of Technology, Newark, NJ, in 1997, all in electrical engineering. From July 1997 to August 2001, he was with Lucent Technologies, New Jersey. He joined the School of Electrical Engineering and Computer Science at Oregon State University in September 2001, and is currently an Associate Professor. His research interests include ultra-wideband schemes, MIMO OFDM systems, communication techniques for multiuser time-varying environments with applications to cellular and indoor wireless communications. He currently serves as an Associate Editor for the IEEE Transactions on Vehicular Technology and IEEE Communications Letters. 